\title{
Competitive Communication Spectrum Economy and Equilibrium
}

\author{
Yinyu Ye
}

Received: 22 October 2013 / Accepted: 26 October 2013 / Published online: 28 November 2013

(C) The Author(s) 2013. This article is published with open access at Springerlink.com

\begin{abstract}
Consider a competitive "spectrum economy" in a communication system where multiple users share a common frequency band and each of them, equipped with an endowed "monetary" budget, will "purchase" its own transmit power spectrum (taking others as given) in maximizing its Shannon utility or pay-off function that includes the effects of interference and subjects to its budget constraint. A market equilibrium is a price spectrum and a frequency power allocation that independently and simultaneously maximizes each user's utility. Furthermore, under an equilibrium the market clears, meaning that the total power demand equals the power supply for every user and every frequency. We prove that such an equilibrium always exists for a discretized version of the problem, and, under a weak-interference condition or the Frequency Division Multiple Access (FMDA) policy, the equilibrium can be computed in polynomial time. This model may lead to an efficient decentralized method for spectrum allocation management and optimization in achieving both higher social utilization and better individual satisfaction. Furthermore, we consider a trading market among individual users to exchange their endowed power spectrum under a price mechanism, and we show that the market price equilibrium also exists and it may lead to a more socially desired spectrum allocation.
\end{abstract}

Keywords Spectrum management · Competitive economy equilibrium · Convex optimization · Complementarity

This work was supported by National Science Foundation Grants (Nos. DMS-0604513 and GOALI 0800151), and Air Force Office of Scientific Research Grant (No. FA9550-09-1-0306).

Y. Ye (凶)

Department of Management Science and Engineering, Stanford University, Stanford, CA 94305, USA

e-mail: yinyu-ye@stanford.edu 


\section{Introduction}

Consider a communication system where multiple users share a common frequency band such as cognitive radio (e.g., [15]) or Digital Subscribe Lines (DSL, e.g., [23]), where interference mitigation is a major design and management concern. A standard approach to eliminate multi-user interference is to divide the available spectrum into multiple tones (or bands) and pre-assign them to the users on a non-overlapping basis, called Frequency Division Multiple Access (FDMA) policy. Although such an approach is well-suited for high speed structured communication in which quality of service is a major concern, it can lead to high system overhead and low bandwidth utilization. With the proliferation of various radio devices and services, multiple wireless systems sharing a common spectrum must coexist [15], and we are naturally led to a situation whereby users can dynamically adjust their transmit power spectral densities over the entire shared spectrum, potentially achieving significantly higher overall throughput and fairness. For such a multi-user system, each user's performance, measured by a Shannon utility function, depends not only on the power allocation (across spectrum) of its own, but also those of other users in the system.

Thus, the dynamic spectrum management problem has recently become a topic of intensive research in the signal processing and digital communication community. From the optimization perspective, the problem solution can be formulated either as a noncooperative Nash game [7, 18, 25, 28]; or as a cooperative utility maximization problem [3, 30]. Several algorithms were proposed to compute a Nash equilibrium solution (Iterative Waterfilling method (IWFA) [7, 28]); or globally optimal power allocations - the dual decomposition method [4, 17, 29] for the cooperative game. Due to the problem's non-convex nature, these algorithms either lack global convergence or may converge to a poor spectrum sharing strategy.

In an attempt to analyze the performance of the dual decomposition algorithms, $\mathrm{Yu}$ and Lui [29] studied the duality gap of the continuous sum-rate maximization problem and showed it to be zero in the general frequency selective case based on engineering intuition. In two recent papers [19, 20], Luo and Zhang presented a systematic study of the dynamic spectrum management problem, covering two key theoretical aspects: complexity and duality. Specifically, they determined the complexity status of the spectrum management problem under various practical settings as well as different choices of system utility functions, and identify subclasses which are polynomial time solvable. In so doing, they clearly delineated the set of computationally tractable problems within the general class of NP-hard spectrum management problems. Furthermore, they rigorously established the zero-duality gap result of $\mathrm{Yu}$ and Lui for the continuous formulation when the interference channels are frequency selective. The asymptotic strong zero-duality result of $[19,20]$ suggests that the Lagrangian dual decomposition approach $[4,17,29]$ may be a viable way to reach approximate optimality for finely discretized spectrum management problems. In fact, when restricted to the FDMA policy, they showed that the Lagrangian dual relaxation, combined with a linear programming scheme, could generate an $\epsilon$-optimal solution for the continuous formulation of the spectrum management problem in polynomial time for any fixed $\epsilon>0$.

Besides computational difficulty, there remain other issues in the Nash equilibrium or the aggregate social utility maximization model. The Nash equilibrium solution 
may not achieve social communication economic efficiency; and, on the other hand, an aggregate optimal power allocation may not simultaneously optimize each user's individual utility. Thus, we naturally turn to a competitive economy equilibrium solution for dynamic spectrum management, where both social economic efficiency and individual optimality could be achieved.

The study of competitive economy equilibria occupies a central place in mathematical economics. This study was formally started by Walras [24] over 100 years ago. In this problem everyone in a population of $n$ agents has an initial endowment of divisible goods or budget and a utility function for consuming all goods - their own and others'. Every agent sells the entire initial endowment and then uses the revenue to buy a bundle of goods such that his or her utility function is maximized (individual optimality) and the market has neither shortage nor surplus (economic efficiency). Walras asked whether prices could be set for every good such that this is possible. An answer was given by Arrow and Debreu in 1954 [1] who showed that such an equilibrium would exist, under very mild conditions, if the utility functions were concave. Their proof was non-constructive and did not offer any algorithm to find such equilibrium prices.

Fisher was the first to consider an algorithm to compute equilibrium prices for a related and different model where agents are divided into two sets: producers and consumers; see Brainard and Scarf [2, 22]. Consumers spend money only to buy goods and maximize their individual utility functions of goods; producers sell their goods only for money. An equilibrium is an assignment of prices to goods so that when every consumer buys a maximal bundle of goods then the market clears, meaning that all the money is spent and all the goods are sold. Fisher's model is a special case of Walras' model when money is also considered a good so that Arrow and Debreu's result applies.

For certain utility functions, the equilibrium problem is actually a social utility maximization problem. For example, Eisenberg and Gale $[12,14]$ give a convex programming (or optimization) formulation whose solution yields equilibriums for the Fisher market with linear utility functions, and Eisenberg [13] extended this approach to derive a convex program for general concave and homogeneous functions of degree 1. Their program consists of maximizing an aggregate social utility function of all consumers over a convex polyhedron defined by supply-demand linear constraints. The Lagrange or dual multipliers of these constraints yield equilibrium prices. Thus, finding a Fisher equilibrium becomes solving a convex optimization problem, and it could be computed by the Ellipsoid method or by efficient interior-point methods in polynomial time. Here, polynomial time means that one can compute an $\epsilon$ approximate equilibrium in a number of arithmetic operations bounded by polynomial in $n$ and $\log \frac{1}{\epsilon}$; or, if there is a rational equilibrium solution, one can compute an exact equilibrium in a number of arithmetic operations bounded by polynomial in $n$ and $L$, where $L$ is the bit-length of the input data, see, e.g., [16]. When the utility functions are linear, the current best arithmetic operations complexity bound is $O\left(\sqrt{m n}(m+n)^{3} L\right)$ given by [26]. Negative results were also obtained for other utilities, see, e.g., Codenotti et al. [8, 27].

However, little is known on the computational complexity for competitive market equilibria with non-homogeneous utility functions $[5,6,11]$, or utility functions 
that include goods purchased by other agents, which is the case in dynamic spectrum management. In the original paper of Arrow and Debreu [1], each user's utility function was described as a function of his or her own actions. Our paper will study the existence and complexity of an equilibrium point that is characterized by the property that each individual is maximizing the pay-off to him or her by controlling his or her own actions, given the actions of the other agents, over the set of actions permitted him or her also in view of the other agents' actions.

We prove in this paper the following.

1. A competitive equilibrium always exists for the communication spectrum market with the Shannon utility for spectrum users and profit utility for the spectrum power provider.

2. Under an additional weak-interference and fixed supply condition, such equilibria form a convex or log-convex set and one can be computed in polynomial time.

3. Under the FMDA policy, the equilibrium is unique and can be computed in polynomial time.

\section{Mathematical Notations}

First, a few mathematical notations. Let $\mathbb{R}^{n}$ denote the $n$-dimensional Euclidean space; $\mathbb{R}_{+}^{n}$ denote the subset of $\mathbb{R}^{n}$ where each coordinate is non-negative. $\mathbb{R}$ and $\mathbb{R}_{+}$ denote the set of real numbers and the set of non-negative real numbers, respectively.

Let $X \in \mathbb{R}_{+}^{m n}$ denote the set of ordered $m$-tuples $X=\left(\boldsymbol{x}_{1}, \cdots, \boldsymbol{x}_{m}\right)$ and let $\bar{X}_{i} \in$ $\mathbb{R}_{+}^{(m-1) n}$ denote the set of ordered $(m-1)$-tuples $X=\left(\boldsymbol{x}_{1}, \cdots, \boldsymbol{x}_{i-1}, \boldsymbol{x}_{i+1}, \cdots, \boldsymbol{x}_{m}\right)$, where $\boldsymbol{x}_{i}=\left(x_{i 1}, \cdots, x_{i n}\right) \in X_{i} \subset \mathbb{R}_{+}^{n}$ for $i=1, \cdots, m$. For each $i$, suppose there is a real utility function $u_{i}$, defined over $X$. Let $A_{i}\left(\overline{\boldsymbol{x}}_{i}\right)$ be a subset of $X_{i}$ defining for each point $\overline{\boldsymbol{x}}_{i} \in \bar{X}_{i}$, Then the sequence $\left[X_{1}, \cdots, X_{m}, u_{1}, \cdots, u_{m}, A_{1}\left(\overline{\boldsymbol{x}}_{1}\right), \cdots, A_{m}\left(\overline{\boldsymbol{x}}_{m}\right)\right]$ will be termed an abstract economy. Here $A_{i}\left(\overline{\boldsymbol{x}}_{i}\right)$ represent the feasible action set of agent $i$ that is possibly restricted by the actions of others, such as the budget restraint that the cost of the goods chosen at current prices not exceed his income, and the prices and possibly some or all of the components of his income are determined by choices made by other agents. Similarly, the utility function $u_{i}\left(\boldsymbol{x}_{i}, \overline{\boldsymbol{x}}_{i}\right)$ for agent $i$ depends on his or her actions $\boldsymbol{x}_{i}$, as well as actions $\overline{\boldsymbol{x}}_{i}$ made by all other agents. Also, denote $\boldsymbol{x}_{j}=\left(x_{1 j}, \cdots, x_{m j}\right) \in \mathbb{R}^{m}$ for a given $\boldsymbol{x} \in X$.

A function $u: \mathbb{R}_{+}^{n} \rightarrow \mathbb{R}_{+}$is said to be concave if for any $\boldsymbol{x}, \boldsymbol{y} \in \mathbb{R}_{+}^{n}$ and any $0 \leqslant$ $\alpha \leqslant 1$, we have $u(\alpha \boldsymbol{x}+(1-\alpha) \boldsymbol{y}) \geqslant \alpha u(\boldsymbol{x})+(1-\alpha) u(\boldsymbol{y})$; and it is strictly concave if $u(\alpha \boldsymbol{x}+(1-\alpha) \boldsymbol{y})>\alpha u(\boldsymbol{x})+(1-\alpha) u(\boldsymbol{y})$ for $0<\alpha<1$. It is monotone increasing if for any $\boldsymbol{x}, \boldsymbol{y} \in \mathbb{R}_{+}^{n}, \boldsymbol{x} \geqslant \boldsymbol{y}$ implies that $u(\boldsymbol{x}) \geqslant u(\boldsymbol{y})$. It is homogeneous of degree $d$ if for any $\boldsymbol{x} \in \mathbb{R}_{+}^{n}$ and any $\alpha>0, u(\alpha \boldsymbol{x})=\alpha^{d} u(\boldsymbol{x})$.

\section{Competitive Communication Spectrum Market}

Let the multi-user communication system consist of $m$ transmitter-receiver pairs sharing a common frequency band $f \in \Omega$. For simplicity, we will call each of such transmitter-receiver pair a "User". Upon normalization, we can assume $\Omega$ to be the 
unit interval [ $\left[\begin{array}{ll}0 & 1\end{array}\right]$. Each user $i$ will be endowed a "monetary" budget $w_{i}>0$ and use it to "purchase or exchange" for power spectral density, $x_{i}(f)$, across $f \in \Omega$, from an open market so as to maximize its own utility $u_{i}\left(x_{i}(f \in \Omega), \bar{x}_{i}(f \in \Omega)\right)$, where $\bar{x}_{i}(f \in \Omega)$ represent power spectral densities obtained by all other users. $w_{i}$ may represent non-real money like a coupon. In some traffic flow applications, they represent "toll" budgets for users to pay toll routes. In other applications, $w_{i}$ simply represents the "importance" weight of certain users; e.g., $w_{i}=1$ for all $i$ means that all users are treated uniformly important. One can also adjust $w_{i}$ to maximize certain aggregate social utility.

There is a second type of agents, called power capacity "Producer or Provider", who installs power capacity spectral density $s(f \in \Omega) \geqslant 0$ to the market from a convex and compact set $S$ to maximize his or her utility.

The third agent, "Market", sets the power spectral unit "price" density $p(f \in$ $\Omega) \geqslant 0 . p(f)$ can be interpreted as a "preference or ranking" spectral density of $f$. For example, $p\left(f_{1}\right)=1$ and $p\left(f_{2}\right)=2$ simply mean that users can use one unit of $s\left(f_{2}\right)$ to trade for two units of $s\left(f_{1}\right)$. In traffic flow applications, $p(f)$ represents the toll fee for route $f$.

Then, User $i$ 's $(i=1, \cdots, m)$ individual utility maximization problem is

$$
\begin{array}{ll}
\underset{x_{i}(f \in \Omega)}{\operatorname{maximize}} & u_{i}\left(x_{i}(f \in \Omega), \bar{x}_{i}(f \in \Omega)\right) \\
\text { subject to } & \int_{\Omega} p(f) x_{i}(f) d f \leqslant w_{i} \\
& x_{i}(f \in \Omega) \geqslant 0 ;
\end{array}
$$

that is, the total payment of "purchased" power spectral density does not exceed his or her endowed budget $w_{i}$.

A commonly recognized utility for user $i, i=1, \cdots, m$, in communication is the Shannon utility [9]:

$$
u_{i}\left(x_{i}(f \in \Omega), \bar{x}_{i}(f \in \Omega)\right)=\int_{\Omega} \log \left(1+\frac{x_{i}(f)}{\sigma_{i}(f)+\sum_{k \neq i} a_{k}^{i}(f) x_{k}(f)}\right) d f
$$

where $\sigma_{i}(f)$ denotes the normalized background noise power for user $i$ at frequency $f$, and $a_{k}^{i}(f)$ is the normalized crosstalk ratio from user $k$ to user $i$ at frequency $f$. Due to normalization we have $a_{i}^{i}(f)=1$ for all $i$ and $f \in \Omega$. One may also subtract the physical cost of total "purchased" power spectral density from the Shannon utility:

$$
\begin{aligned}
& u_{i}\left(x_{i}(f \in \Omega), \bar{x}_{i}(f \in \Omega)\right) \\
& \quad=\int_{\Omega} \log \left(1+\frac{x_{i}(f)}{\sigma_{i}(f)+\sum_{k \neq i} a_{k}^{i}(f) x_{k}(f)}\right) d f-c_{i} \int_{\Omega} x_{i}(f) d f,
\end{aligned}
$$

where $c_{i}$ is a constant cost rate for user $i$.

The power provider's individual utility maximization problem is

$$
\begin{array}{ll}
\underset{s(f \in \Omega)}{\operatorname{maximize}} & u_{S}(s(f \in \Omega), p(f \in \Omega)) \\
\text { subject to } & s(f \in \Omega) \in S,
\end{array}
$$


where $u_{s}(s(f \in \Omega), p(f \in \Omega))$ represents the utility function of the power capacity provider who installs $s(f \in \Omega)$ and $S$ is a physical feasible set (can be a fixed point). For example,

$$
u_{s}(s(f \in \Omega), p(f \in \Omega))=\int_{\Omega} p(f) s(f) d f-c(s(f \in \Omega))
$$

that is, the profit made by installing power spectral density $s(f)$ where $c(s(f \in \Omega))$ is a cost function.

A competitive market equilibrium is a density point $\left[x_{1}^{*}(f), \cdots, x_{m}^{*}(f), s^{*}(f)\right.$, $\left.p^{*}(f)\right], f \in \Omega$ such that we have the following.

- (User optimality) $x_{i}^{*}(f \in \Omega)$ is a maximizer of (3.1) given $\bar{x}_{i}^{*}(f \in \Omega)$ and $p^{*}(f \in$ $\Omega$ ) for every $i$.

- (Producer optimality) $s^{*}(f \in \Omega)$ is a maximizer of (3.3) given $p^{*}(f \in \Omega)$.

- (Market efficiency) $p(f \in \Omega) \geqslant 0, \sum_{i=1}^{m} x_{i}^{*}(f) \leqslant s^{*}(f), \quad p^{*}(f)\left(s^{*}(f)-\right.$ $\left.\sum_{i=1}^{m} x_{i}^{*}(f)\right)=0, \forall f \in \Omega$.

The last condition says that if power capacity $s^{*}(f)$ is greater than the needed density, $\sum_{i=1}^{m} x_{i}^{*}(f)$, at frequency $f$, then its equilibrium price density $p^{*}(f)=0$.

We remark that this is a rather artificial exchange or trading open market, where the price $p$ and budget $w$ do not necessarily have physical interpretations. The meaningful outputs may be only those power spectrum assignments, and $w$ and $p$ are economic mechanisms mainly used to influence users' behavior over the communication channel selection to achieve a larger market utilization.

In reality, the frequency band range $\Omega$ is discretized by $n$ tones: $\Omega=\left\{f_{1}, f_{2}\right.$, $\left.\cdots, f_{n}\right\}$, where the Shannon utility for user $i, i=1, \cdots, m$, becomes

$$
u_{i}\left(\boldsymbol{x}_{i}, \overline{\boldsymbol{x}}_{i}\right)=\sum_{j=1}^{n} \log \left(1+\frac{x_{i j}}{\sigma_{i j}+\sum_{k \neq i} a_{k j}^{i} x_{k j}}\right)
$$

where variable $\boldsymbol{x}_{i}=\left(x_{i 1}, \cdots, x_{i n}\right) \in \mathbb{R}_{+}^{n}$ and $x_{i j}$ is the power units purchased by User $i$ for tone $j$, variables in $\overline{\boldsymbol{x}}_{i} \in \mathbb{R}_{+}^{(m-1) n}$ are power units purchased by all other users, parameter $\sigma_{i j}$ denotes the normalized background noise power for user $i$ at tone $j$, and parameter $a_{k j}^{i}$ is the normalized crosstalk ratio from user $k$ to user $i$ at tone $j$. Due to normalization we have $a_{i j}^{i}=1$ for all $i, j$. Clearly, $u_{i}\left(\boldsymbol{x}_{i}, \overline{\boldsymbol{x}}_{i}\right)$ is a continuous concave and monotone increasing function in $\boldsymbol{x}_{i} \in \mathbb{R}_{+}^{n}$ for every $\overline{\boldsymbol{x}}_{i} \in \mathbb{R}_{+}^{(m-1) n}$. Again, one may also adjust the utility function to

$$
u_{i}\left(\boldsymbol{x}_{i}, \overline{\boldsymbol{x}}_{i}\right)=\sum_{j=1}^{n} \log \left(1+\frac{x_{i j}}{\sigma_{i j}+\sum_{k \neq i} a_{k j}^{i} x_{k j}}\right)-c_{i}\left(\sum_{j=1}^{n} x_{i j}\right),
$$

that is, subtracting a physical cost of total "purchased" tone powers from the Shannon utility.

Then, a competitive communication spectrum market equilibrium is a point of $\left[\boldsymbol{x}_{1}^{*}, \cdots, \boldsymbol{x}_{m}^{*}, \boldsymbol{s}^{*}, \boldsymbol{p}^{*}\right]$, where $\boldsymbol{s}^{*}=\left(s_{1}^{*}, \cdots, s_{n}^{*}\right) \in \mathbb{R}_{+}^{n}$ and $s_{j}^{*}$ is the total power capacity units installed by the second power provider, and $\boldsymbol{p}^{*}=\left(p_{1}^{*}, \cdots, p_{n}^{*}\right) \in \mathbb{R}_{+}^{n}$ and $p_{j}^{*}$ is the unit price for tone $j$ set by Market; such that we have the following. 
1. (User optimality) $\boldsymbol{x}_{i}^{*}, i=1, \cdots, m$, is a maximizer of

$$
\begin{array}{cl}
\underset{\boldsymbol{x}_{i}}{\operatorname{maximize}} & u_{i}\left(\boldsymbol{x}_{i}, \overline{\boldsymbol{x}}_{i}^{*}\right) \\
\text { subject to } & \sum_{j=1}^{n} p_{j}^{*} x_{i j} \leqslant w_{i} \\
& \boldsymbol{x}_{i} \in X_{i} \subset \mathbb{R}_{+}^{n},
\end{array}
$$

where $\overline{\boldsymbol{x}}_{i}^{*}=\left[\boldsymbol{x}_{1}^{*}, \cdots, \boldsymbol{x}_{i-1}^{*}, \boldsymbol{x}_{i+1}^{*}, \cdots, \boldsymbol{x}_{n}^{*}\right]$ and $X_{i}$ is a physical feasible set. For example,

$$
X_{i}=\left\{\boldsymbol{x}_{i} \in \mathbb{R}_{+}^{n}: \sum_{j=1}^{n} x_{i j} \leqslant b_{i}\right\}
$$

where $b_{i}>0$ is a physical battery power budget for user $i$.

2. (Producer optimality) $s^{*}$ is a maximizer of

$$
\begin{array}{ll}
\underset{s}{\operatorname{maximize}} & u_{s}\left(\boldsymbol{s}, \boldsymbol{p}^{*}\right) \\
\text { subject to } & \boldsymbol{s} \in S \subset \mathbb{R}_{+}^{n} .
\end{array}
$$

3. (Market efficiency) $\boldsymbol{p}^{*} \geqslant \mathbf{0}, \sum_{i=1}^{m} x_{i j}^{*} \leqslant \boldsymbol{s}_{j}^{*}, p_{j}^{*}\left(s_{j}^{*}-\sum_{i=1}^{m} x_{i j}^{*}\right)=0$ for all $j$.

The following theorem directly follows Arrow and Debreu [1].

Theorem 3.1 Let $u_{i}\left(\boldsymbol{x}_{i}, \overline{\boldsymbol{x}}_{i}\right)$ be a continuous and concave function in $\boldsymbol{x}_{i} \in \mathbb{R}_{+}^{n}$ for every $\overline{\boldsymbol{x}}_{i} \in \mathbb{R}_{+}^{(m-1) n}, u_{s}(\boldsymbol{s}, \boldsymbol{p})$ be a continuous and concave function in $\boldsymbol{s}$ for every $\boldsymbol{p}$, $X_{i} \subset \mathbb{R}_{+}^{n}, i=1, \cdots, m$, and $S \subset \mathbb{R}_{+}^{n}$ be closed convex sets, and $S$ be furthermore bounded. Then, the discretized communication spectrum market has a competitive equilibrium.

The proof of the theorem is identical to the one given in [1] based on the Lemma of Abstract Economy developed by Debreu [10] and Nash [21], where money is a good whose price is normalized as 1 . The only difference is that in [1], the utility function $u_{i}$ is a function of $\boldsymbol{x}_{i}$ and nothing else. However, the Lemma of Abstract Economy actually allows agent $i$ 's utility $u_{i}$ to be a function of $\boldsymbol{x}_{i}$, the action made by agent $i$, and $\overline{\boldsymbol{x}}_{i}$, the actions made by all other agents.

Furthermore, if $\boldsymbol{p}$ and $\boldsymbol{s}$ are fixed and only Users are the agents in the game, the equilibrium problem reduces to a Nash equilibrium problem. By allowing $\boldsymbol{p}$ and $\boldsymbol{s}$ vary in the game, we hope to potentially achieve a more efficient spectrum economy.

Since the Shannon utility function of (3.4) is continuous, concave and monotone increasing in $\boldsymbol{x}_{i} \in \mathbb{R}_{+}^{n}$ for every $\overline{\boldsymbol{x}}_{i} \in \mathbb{R}_{+}^{(m-1) n}$. Our main result is the following corollary.

Corollary 3.1 Let the power capacity provider utility $u_{s}(\boldsymbol{s}, \boldsymbol{p})$ be a continuous and concave function in $\mathbf{s}$ for every $\boldsymbol{p}$, and $X_{i} \subset \mathbb{R}_{+}^{n}, i=1, \cdots, m$, and $S \subset \mathbb{R}_{+}^{n}$ be closed convex sets, and $S$ be furthermore bounded. Then, the discretized communication spectrum market with the Shannon utility has a competitive equilibrium. 


\section{Equilibrium Characterization}

For simplicity, let the power capacity set $S$ is represented by a polyhedron

$$
S=\{\boldsymbol{s}: B \boldsymbol{s} \leqslant \boldsymbol{r}, \boldsymbol{s} \geqslant \mathbf{0}\}
$$

for given constraint matrix $B$ and resource vector $\boldsymbol{r}$, and let the power supply cost function be linear $c(s)=c^{T}$ f for a given cost coefficient vector $c$. Then, the optimality function of (3.6) is such that there is $y^{*}$ such that

$$
\begin{aligned}
B \boldsymbol{s}^{*} & \leqslant \boldsymbol{r}, \\
\boldsymbol{p}^{*}-\boldsymbol{c} & \leqslant B^{T} \boldsymbol{y}^{*}, \\
\boldsymbol{r}^{T} \boldsymbol{y}^{*} & =\left(\boldsymbol{p}^{*}-c\right)^{T} \boldsymbol{s}^{*}, \\
\boldsymbol{s}^{*}, \boldsymbol{y}^{*} & \geqslant \mathbf{0} .
\end{aligned}
$$

Now consider the optimality conditions of (3.5) where, for simplicity, we let $X_{i}=\mathbb{R}_{+}^{n}$. They are

$$
\begin{aligned}
w_{i} \cdot \nabla_{\boldsymbol{x}_{i}} u_{i}\left(\boldsymbol{x}_{i}^{*}, \overline{\boldsymbol{x}}_{i}^{*}\right) & \leqslant\left(\nabla_{\boldsymbol{x}_{i}} u_{i}\left(\boldsymbol{x}_{i}^{*}, \overline{\boldsymbol{x}}_{i}^{*}\right)^{T} \boldsymbol{x}_{i}^{*}\right) \cdot \boldsymbol{p}^{*}, \\
\left(\boldsymbol{p}^{*}\right)^{T} \boldsymbol{x}_{i}^{*} & =w_{i}, \\
\boldsymbol{x}_{i}^{*} & \geqslant \mathbf{0},
\end{aligned}
$$

where $\nabla_{\boldsymbol{x}_{i}} u\left(\boldsymbol{x}_{i}, \overline{\boldsymbol{x}}_{i}\right) \in \mathbb{R}^{n}$ denotes any sub-gradient vector of $u\left(\boldsymbol{x}_{i}, \overline{\boldsymbol{x}}_{i}\right)$ with respect to $\boldsymbol{x}_{i}$.

The complete necessary and sufficient conditions for a competitive equilibrium can be summarized as:

$$
\begin{aligned}
w_{i} \cdot \nabla_{\boldsymbol{x}_{i}} u_{i}\left(\boldsymbol{x}_{i}^{*}, \overline{\boldsymbol{x}}_{i}^{*}\right) & \leqslant\left(\nabla_{\boldsymbol{x}_{i}} u_{i}\left(\boldsymbol{x}_{i}^{*}, \overline{\boldsymbol{x}}_{i}^{*}\right)^{T} \boldsymbol{x}_{i}^{*}\right) \cdot \boldsymbol{p}^{*}, \quad \forall i \\
B \boldsymbol{s}^{*} & \leqslant \boldsymbol{r}, \\
\boldsymbol{p}^{*}-\boldsymbol{c} & \leqslant B^{T} \boldsymbol{y}^{*}, \\
\sum_{i} \boldsymbol{x}_{i}^{*} & \leqslant \boldsymbol{s}^{*}, \\
\boldsymbol{c}^{T} \boldsymbol{s}^{*}+\boldsymbol{r}^{T} \boldsymbol{y}^{*} & \leqslant \sum_{i} w_{i}, \\
\boldsymbol{x}_{i}^{*}, \boldsymbol{p}^{*}, \boldsymbol{s}^{*}, \boldsymbol{y}^{*} & \geqslant \mathbf{0}, \quad \forall i .
\end{aligned}
$$

Note that the conditions $\left(\boldsymbol{p}^{*}\right)^{T} \boldsymbol{s}^{*}=\boldsymbol{c}^{T} \boldsymbol{s}^{*}+\boldsymbol{r}^{T} \boldsymbol{y}^{*}$ and $\left(\boldsymbol{p}^{*}\right)^{T} \boldsymbol{x}_{i}^{*}=w_{i}$ for all $i$ are implied by the conditions in (4.3): multiplying $\boldsymbol{x}_{i}^{*} \geqslant \mathbf{0}$ to both sides of the first inequality, $\boldsymbol{y}^{*} \geqslant \mathbf{0}$ to both sides of the second inequality, $\boldsymbol{s}^{*} \geqslant \mathbf{0}$ to both sides of the third inequality in (4.3), we have $\left(\boldsymbol{p}^{*}\right)^{T} \boldsymbol{x}_{i}^{*} \geqslant w_{i}$ for all $i, \boldsymbol{r}^{T} \boldsymbol{y}^{*} \geqslant\left(\boldsymbol{s}^{*}\right)^{T} B^{T} \boldsymbol{y}^{*}$, and $\left(s^{*}\right)^{T} B^{T} y^{*} \geqslant\left(p^{*}-c\right)^{T} s^{*}$, which, together with other inequality conditions in (4.3), imply

$$
\sum_{i} w_{i} \geqslant \boldsymbol{c}^{T} \boldsymbol{s}^{*}+\boldsymbol{r}^{T} \boldsymbol{y}^{*} \geqslant\left(\boldsymbol{p}^{*}\right)^{T} \boldsymbol{s}^{*} \geqslant\left(\boldsymbol{p}^{*}\right)^{T}\left(\sum_{i} \boldsymbol{x}_{i}^{*}\right)=\sum_{i}\left(\boldsymbol{p}^{*}\right)^{T} \boldsymbol{x}_{i}^{*} \geqslant \sum_{i} w_{i},
$$

that is, every inequality in the sequence must be tight, which implies $\left(p^{*}\right)^{T} s^{*}=$ $c^{T} \boldsymbol{s}^{*}+\boldsymbol{r}^{T} \boldsymbol{y}^{*},\left(\boldsymbol{p}^{*}\right)^{T}\left(\sum_{i} \boldsymbol{x}_{i}^{*}\right)=\left(\boldsymbol{p}^{*}\right)^{T} \boldsymbol{s}^{*}$, and $\left(\boldsymbol{p}^{*}\right)^{T} \boldsymbol{x}_{i}^{*}=w_{i}$ for all $i$.

Thus, we have a characterization theorem of a competitive equilibrium. 
Theorem 4.1 Every equilibrium of the discretized communication spectrum market with the Shannon utility has the following properties:

1. $\boldsymbol{p}^{*}>\mathbf{0}$ (every tone power has a price);

2. $\sum_{i} \boldsymbol{x}_{i}^{*}=\boldsymbol{s}^{*}$ (produce what is needed);

3. $\left(\boldsymbol{p}^{*}\right)^{T} \boldsymbol{s}^{*}=\sum_{i} w_{i}$ (all user budgets go to the provider);

4. If $x_{i j}^{*}>0$ then $\left(\nabla_{\boldsymbol{x}_{i}} u_{i}\left(\boldsymbol{x}_{i}^{*}, \overline{\boldsymbol{x}}_{i}^{*}\right)^{T} \boldsymbol{x}_{i}^{*}\right) \cdot p_{j}^{*}-w_{i} \cdot\left(\nabla_{\boldsymbol{x}_{i}} u_{i}\left(\boldsymbol{x}_{i}^{*}, \overline{\boldsymbol{x}}_{i}^{*}\right)\right)_{j}=0$ for all $i, j$ (every user only purchases most valuable tone power).

Proof Note that

$$
\left(\nabla_{\boldsymbol{x}_{i}} u_{i}\left(\boldsymbol{x}_{i}, \overline{\boldsymbol{x}}_{i}\right)\right)_{j}=\frac{1}{\sigma_{i j}+\sum_{k \neq i} a_{k j}^{i} x_{k j}+x_{i j}}>0, \quad \forall \boldsymbol{x} \geqslant \mathbf{0} .
$$

Thus,

$$
w_{i} \cdot \nabla_{\boldsymbol{x}_{i}} u_{i}\left(\boldsymbol{x}_{i}^{*}, \overline{\boldsymbol{x}}_{i}^{*}\right)>\mathbf{0},
$$

so that the first inequality of (4.3) implies that $\boldsymbol{p}^{*}>\mathbf{0}$.

The second property is from $\left(\boldsymbol{p}^{*}\right)^{T}\left(\sum_{i} \boldsymbol{x}_{i}^{*}\right)=\left(\boldsymbol{p}^{*}\right)^{T} \boldsymbol{s}^{*}, \sum_{i} \boldsymbol{x}_{i}^{*} \leqslant \boldsymbol{s}^{*}$ and $\boldsymbol{p}^{*}>\mathbf{0}$.

The third is from $\left(\boldsymbol{p}^{*}\right)^{T} \boldsymbol{x}_{i}^{*}=w_{i}$ for all $i$ and $\sum_{i} \boldsymbol{x}_{i}^{*}=\boldsymbol{s}^{*}$.

The last one is from the complementarity condition of user optimality.

\section{Equilibrium for a Weak-Interference Market}

The inequalities and equalities in (4.3) are all linear, except the first

$$
w_{i} \cdot \nabla_{\boldsymbol{x}_{i}} u_{i}\left(\boldsymbol{x}_{i}^{*}, \overline{\boldsymbol{x}}_{i}^{*}\right) \leqslant\left(\nabla_{\boldsymbol{x}_{i}} u_{i}\left(\boldsymbol{x}_{i}^{*}, \overline{\boldsymbol{x}}_{i}^{*}\right)^{T} \boldsymbol{x}_{i}^{*}\right) \cdot \boldsymbol{p}^{*} .
$$

Now we consider a weak-interference communication channel where the Shannon utility function is approximated by

$$
u_{i}\left(\boldsymbol{x}_{i}, \overline{\boldsymbol{x}}_{i}\right)=\sum_{j=1}^{n} \log \left(1+\frac{x_{i j}}{\sigma_{i j}+a_{j}^{i}\left(\sum_{k \neq i} x_{k j}\right)}\right)
$$

where $a_{j}^{i}$ represent the average of normalized crosstalk ratios for $k \neq i$. Furthermore, we assume $0 \leqslant a_{j}^{i} \leqslant 1$, that is, the average cross-interference ratio is not above 1 or it is less than the self-interference ratio (always normalized to 1 ); and $S=\left\{s^{*}\right\}$ is a singleton, that is, the power supply $\boldsymbol{s}^{*}$ is fixed.

The partial derivative of $u_{i}\left(\boldsymbol{x}_{i}, \overline{\boldsymbol{x}}_{i}\right)$ to $x_{i j}$ is

$$
\left(\nabla_{\boldsymbol{x}_{i}} u_{i}\left(\boldsymbol{x}_{i}, \overline{\boldsymbol{x}}_{i}\right)\right)_{j}=\frac{1}{\sigma_{i j}+a_{j}^{i}\left(\sum_{k \neq i} x_{k j}\right)+x_{i j}}, \quad \forall j
$$

so that

$$
\nabla_{\boldsymbol{x}_{i}} u_{i}\left(\boldsymbol{x}_{i}, \overline{\boldsymbol{x}}_{i}\right)^{T} \boldsymbol{x}_{i}=\sum_{j=1}^{n} \frac{x_{i j}}{\sigma_{i j}+a_{j}^{i}\left(\sum_{k \neq i} x_{k j}\right)+x_{i j}} .
$$


At an equilibrium characterized by (4.3),

$$
\sum_{k \neq i} x_{k j}^{*}=s_{j}^{*}-x_{i j}^{*}, \quad \forall j .
$$

Thus,

$$
\left(\nabla_{\boldsymbol{x}_{i}} u_{i}\left(\boldsymbol{x}_{i}^{*}, \overline{\boldsymbol{x}}_{i}^{*}\right)\right)_{j}=\frac{1}{\sigma_{i j}+a_{j}^{i} s_{j}^{*}+\left(1-a_{j}^{i}\right) x_{i j}^{*}}, \quad \forall j
$$

so that

$$
\nabla_{\boldsymbol{x}_{i}} u_{i}\left(\boldsymbol{x}_{i}^{*}, \overline{\boldsymbol{x}}_{i}^{*}\right)^{T} \boldsymbol{x}_{i}=\sum_{j=1}^{n} \frac{x_{i j}^{*}}{\sigma_{i j}+a_{j}^{i} s_{j}^{*}+\left(1-a_{j}^{i}\right) x_{i j}^{*}} .
$$

Then, using the logarithmic transformation, one can rewrite the nonlinear inequality in (4.3) as

$$
\begin{aligned}
& \log \left(\sigma_{i j}+a_{j}^{i} s_{j}^{*}+\left(1-a_{j}^{i}\right) x_{i j}^{*}\right)+\log \left(p_{j}^{*}\right)+\log \left(\sum_{j=1}^{n} \frac{x_{i j}^{*}}{\sigma_{i j}+a_{j}^{i} s_{j}^{*}+\left(1-a_{j}^{i}\right) x_{i j}^{*}}\right) \\
& \geqslant \log \left(w_{i}\right), \quad \forall i, j .
\end{aligned}
$$

This is actually a convex inequality, since the function on the left is a (strictly) concave function in $x_{i j}^{*}$ and $p_{j}^{*}$ for any constant $1-a_{j}^{i} \geqslant 0$.

Therefore, we have the following.

Theorem 5.1 Under the weak-interference communication channel and fixed power supply condition, the competitive equilibrium set of the discretized communication spectrum market is convex.

We remark that the cooperative utility maximization approach, with the Shannon utility given by (5.1) and fixed power supply, is still a non-convex optimization problem. However, its equilibrium set is convex!

It is known that the convex inequality (5.2) admits an efficient barrier function (see Deng et al. $[5,11])$, so that we have the following.

Corollary 5.1 An equilibrium of the discretized communication spectrum market under the weak-interference communication channel and fixed power supply condition can be computed in polynomial time.

Complementarity Property 4 of Theorem 4.1 implies

$$
\begin{aligned}
& \log \left(\sigma_{i j}+a_{j}^{i} s_{j}^{*}+\left(1-a_{j}^{i}\right) x_{i j}^{*}\right)+\log \left(p_{j}^{*}\right)+\log \left(\sum_{j=1}^{n} \frac{x_{i j}^{*}}{\sigma_{i j}+a_{j}^{i} s_{j}^{*}+\left(1-a_{j}^{i}\right) x_{i j}^{*}}\right) \\
& =\log \left(w_{i}\right), \quad \forall x_{i j}^{*}>0 .
\end{aligned}
$$

Let $\left[\left(\boldsymbol{x}^{*}\right)^{1},\left(\boldsymbol{p}^{*}\right)^{1}\right]$ and $\left[\left(\boldsymbol{x}^{*}\right)^{2},\left(\boldsymbol{p}^{*}\right)^{2}\right]$ be two distinct equilibrium points, then Theorem 5.1 implies that $\left[0.5\left(\boldsymbol{x}^{*}\right)^{1}+0.5\left(\boldsymbol{x}^{*}\right)^{2}, \boldsymbol{s}^{*}, 0.5\left(\boldsymbol{p}^{*}\right)^{1}+0.5\left(\boldsymbol{p}^{*}\right)^{2}\right]$ is also an equilibrium, so that 


$$
\begin{aligned}
& \log \left(\sigma_{i j}+a_{j}^{i} s_{j}^{*}+\left(1-a_{j}^{i}\right)\left(0.5\left(x^{*}\right)_{i j}^{1}+0.5\left(x^{*}\right)_{i j}^{2}\right)\right)+\log \left(0.5\left(p^{*}\right)_{j}^{1}+0.5\left(p^{*}\right)_{j}^{2}\right) \\
& \quad+\log \left(\sum_{j=1}^{n} \frac{0.5\left(x^{*}\right)_{i j}^{1}+0.5\left(x^{*}\right)_{i j}^{2}}{\sigma_{i j}+a_{j}^{i} s_{j}^{*}+\left(1-a_{j}^{i}\right)\left(0.5\left(x^{*}\right)_{i j}^{1}+0.5\left(x^{*}\right)_{i j}^{2}\right)}\right) \\
& =\log \left(w_{i}\right), \quad \forall \max \left\{\left(x^{*}\right)_{i j}^{1},\left(x^{*}\right)_{i j}^{2}\right\}>0 .
\end{aligned}
$$

However, the function on the left is strictly concave in $\boldsymbol{p}^{*}$, and in $\boldsymbol{x}^{*}$ if $a_{j}^{i}<1$, so that

$$
\begin{aligned}
& \log \left(\sigma_{i j}+a_{j}^{i} s_{j}^{*}+\left(1-a_{j}^{i}\right)\left(0.5\left(x^{*}\right)_{i j}^{1}+0.5\left(x^{*}\right)_{i j}^{2}\right)\right)+\log \left(0.5\left(p^{*}\right)_{j}^{1}+0.5\left(p^{*}\right)_{j}^{2}\right) \\
& \quad+\log \left(\sum_{j=1}^{n} \frac{0.5\left(x^{*}\right)_{i j}^{1}+0.5\left(x^{*}\right)_{i j}^{2}}{\sigma_{i j}+a_{j}^{i} s_{j}^{*}+\left(1-a_{j}^{i}\right)\left(0.5\left(x^{*}\right)_{i j}^{1}+0.5\left(x^{*}\right)_{i j}^{2}\right)}\right) \\
&> 0.5\left(\log \left(\sigma_{i j}+a_{j}^{i} s_{j}^{*}+\left(1-a_{j}^{i}\right)\left(x^{*}\right)_{i j}^{1}\right)+\log \left(\left(p^{*}\right)_{j}^{1}\right)\right. \\
&\left.+\log \left(\sum_{j=1}^{n} \frac{\left(x^{*}\right)_{i j}^{1}}{\sigma_{i j}+a_{j}^{i} s_{j}^{*}+\left(1-a_{j}^{i}\right)\left(x^{*}\right)_{i j}^{1}}\right)\right) \\
&+0.5\left(\log \left(\sigma_{i j}+a_{j}^{i} s_{j}^{*}+\left(1-a_{j}^{i}\right)\left(x^{*}\right)_{i j}^{2}\right)+\log \left(\left(p^{*}\right)_{j}^{2}\right)\right. \\
&\left.+\log \left(\sum_{j=1}^{n} \frac{\left(x^{*}\right)_{i j}^{2}}{\sigma_{i j}+a_{j}^{i} s_{j}^{*}+\left(1-a_{j}^{i}\right)\left(x^{*}\right)_{i j}^{2}}\right)\right) \\
& \geqslant 0.5 \log \left(w_{i}\right)+0.5 \log \left(w_{i}\right)=\log \left(w_{i}\right), \quad \forall \max \left\{\left(x^{*}\right)_{i j}^{1},\left(x^{*}\right)_{i j}^{2}\right\}>0 .
\end{aligned}
$$

Thus, we must have $\left(\boldsymbol{p}^{*}\right)^{1}=\left(\boldsymbol{p}^{*}\right)^{2}>\mathbf{0}$, and $\left(x^{*}\right)_{i j}^{1}=\left(x^{*}\right)_{i j}^{2}, \forall \max \left\{\left(x^{*}\right)_{i j}^{1},\left(x^{*}\right)_{i j}^{2}\right\}>$ 0 if $a_{j}^{i}<1$, which imply that the equilibrium point is unique.

Therefore, we have the following.

Theorem 5.2 Under the weak-interference communication channel and fixed power supply condition, the competitive price equilibrium of the discretized communication spectrum market is unique. Moreover, if the crosstalk ratio $a_{j}^{i}$ is strictly less than 1 , then the power allocation $x_{i j}^{*}$ is also unique.

\section{The Spectrum Trading Market}

Unlike the market described above where each user is equipped with an endowed "monetary" budget, in the spectrum trading market each user is endowed with a bundle of allocated spectrum power, say $\boldsymbol{w}_{i} \in \mathbb{R}^{n}$, where each entry represents the power allocation for user $i$ on channel or tone $j, j=1, \cdots, n$. Now they trade their spectrum power under a market price to maximize their individual utility function. Here, the total spectrum power capacities available on the market are

$$
\overline{\boldsymbol{s}}=\sum_{i=1}^{m} \boldsymbol{w}_{i},
$$


and they are fixed.

A competitive communication spectrum market equilibrium is a point of $\left[x_{1}^{*}, \cdots\right.$, $\left.\boldsymbol{x}_{m}^{*}, \boldsymbol{p}^{*}\right]$, where $\boldsymbol{p}^{*}=\left(p_{1}^{*}, \cdots, p_{n}^{*}\right) \in \mathbb{R}_{+}^{n}$ and $p_{j}^{*}$ is the unit price on tone $j$; such that

1. (User optimality) $\boldsymbol{x}_{i}^{*}, i=1, \cdots, m$, is a maximizer of

$$
\begin{array}{cl}
\underset{\boldsymbol{x}_{i}}{\operatorname{maximize}} & u_{i}\left(\boldsymbol{x}_{i}, \overline{\boldsymbol{x}}_{i}^{*}\right) \\
\text { subject to } & \sum_{j=1}^{n} p_{j}^{*} x_{i j} \leqslant \boldsymbol{w}_{i}^{T} \boldsymbol{p}^{*} \\
& \boldsymbol{x}_{i} \geqslant \mathbf{0} .
\end{array}
$$

2. (Market efficiency) $\boldsymbol{p}^{*} \geqslant \mathbf{0}, \sum_{i=1}^{m} x_{i j}^{*} \leqslant \bar{s}_{j}$ and $p_{j}^{*}\left(\bar{s}_{j}-\sum_{i=1}^{m} x_{i j}^{*}\right)=0$ for all $j$.

Note that (6.1) is homogeneous in $\boldsymbol{p}^{*}$, so that if $\boldsymbol{p}^{*}$ is an equilibrium price vector, so is $\alpha \cdot \boldsymbol{p}^{*}$ for any positive multiplier $\alpha$.

Again, following the theorem of Arrow and Debreu [1], we have the following.

Corollary 6.1 The trading spectrum market with the Shannon utility has a competitive equilibrium.

From the optimality conditions of (6.1):

$$
\begin{aligned}
\left(\boldsymbol{w}_{i}^{T} \boldsymbol{p}^{*}\right) \cdot \nabla_{\boldsymbol{x}_{i}} u_{i}\left(\boldsymbol{x}_{i}^{*}, \overline{\boldsymbol{x}}_{i}^{*}\right) & \leqslant\left(\nabla_{\boldsymbol{x}_{i}} u_{i}\left(\boldsymbol{x}_{i}^{*}, \overline{\boldsymbol{x}}_{i}^{*}\right)^{T} \boldsymbol{x}_{i}^{*}\right) \cdot \boldsymbol{p}^{*}, \\
\left(\boldsymbol{p}^{*}\right)^{T} \boldsymbol{x}_{i}^{*} & =\boldsymbol{w}_{i}^{T} \boldsymbol{p}^{*}, \\
\boldsymbol{x}_{i}^{*} & \geqslant \mathbf{0},
\end{aligned}
$$

we can derive the complete necessary and sufficient conditions for a competitive equilibrium:

$$
\begin{aligned}
\left(\boldsymbol{w}_{i}^{T} \boldsymbol{p}^{*}\right) \cdot \nabla_{\boldsymbol{x}_{i}} u_{i}\left(\boldsymbol{x}_{i}^{*}, \overline{\boldsymbol{x}}_{i}^{*}\right) & \leqslant\left(\nabla_{\boldsymbol{x}_{i}} u_{i}\left(\boldsymbol{x}_{i}^{*}, \overline{\boldsymbol{x}}_{i}^{*}\right)^{T} \boldsymbol{x}_{i}^{*}\right) \cdot \boldsymbol{p}^{*}, \quad \forall i \\
\sum_{i} \boldsymbol{x}_{i}^{*} & \leqslant \overline{\boldsymbol{s}}, \\
\boldsymbol{x}_{i}^{*}, \boldsymbol{p}^{*} & \geqslant \mathbf{0}, \quad \forall i .
\end{aligned}
$$

Note that the condition $\left(\boldsymbol{p}^{*}\right)^{T} \boldsymbol{x}_{i}^{*}=\boldsymbol{w}_{i}^{T} \boldsymbol{p}^{*}$ for all $i$ is implied by the conditions in (6.3): multiplying $\boldsymbol{x}_{i}^{*} \geqslant \mathbf{0}$ to both sides of the first inequality, we have $\left(\boldsymbol{p}^{*}\right)^{T} \boldsymbol{x}_{i}^{*} \geqslant$ $\boldsymbol{w}_{i}^{T} \boldsymbol{p}^{*}$ for all $i$, which imply

$$
\sum_{i} \boldsymbol{w}_{i}^{T} \boldsymbol{p}^{*} \leqslant \sum_{i}\left(\boldsymbol{p}^{*}\right)^{T} \boldsymbol{x}_{i}^{*} \leqslant\left(\boldsymbol{p}^{*}\right)^{T} \overline{\boldsymbol{s}}=\sum_{i} \boldsymbol{w}_{i}^{T} \boldsymbol{p}^{*},
$$

that is, every inequality in the sequence must be tight, which implies $\left(\boldsymbol{p}^{*}\right)^{T} \boldsymbol{x}_{i}^{*}=$ $\boldsymbol{w}_{i}^{T} \boldsymbol{p}^{*}$ for all $i$ and $\sum_{i} \boldsymbol{x}_{i}^{*}=\overline{\boldsymbol{s}}$.

Similarly, we have a characterization theorem of a competitive equilibrium.

Theorem 6.1 Every equilibrium of the trading spectrum market with the Shannon utility has the following properties: 
1. $\boldsymbol{p}^{*}>\mathbf{0}$ (every tone power has a price);

2. $\sum_{i} \boldsymbol{x}_{i}^{*}=\overline{\boldsymbol{s}}$ (all power endowments are traded).

3. If the equilibrium allocation has a property that each tone is occupied by a single user, then the Shannon utility at the equilibrium is improved for every user (trading benefits every one).

The proof of the last statement is based on the fact that, for every $i$,

$$
u_{i}\left(\boldsymbol{x}_{i}^{*}, \mathbf{0}\right) \geqslant u_{i}\left(\boldsymbol{x}_{i}^{*}, \overline{\boldsymbol{w}}_{i}\right) \geqslant u_{i}\left(\boldsymbol{w}_{i}, \overline{\boldsymbol{w}}_{i}\right)
$$

where the first inequality is from that there is no interference at the equilibrium and the second inequality holds since $\boldsymbol{w}_{i}$ is a feasible solution for (6.1) at the corresponding equilibrium price $\boldsymbol{p}^{*}$.

Under the same weak-interference communication channel condition and using the logarithmic transformation, one can rewrite the nonlinear inequality in (6.3) as

$$
\begin{aligned}
& \log \left(\sigma_{i j}+a_{j}^{i} \bar{s}_{j}+\left(1-a_{j}^{i}\right) x_{i j}^{*}\right)+\log \left(p_{j}^{*}\right)+\log \left(\sum_{j=1}^{n} \frac{x_{i j}^{*}}{\sigma_{i j}+a_{j}^{i} \bar{s}_{j}+\left(1-a_{j}^{i}\right) x_{i j}^{*}}\right) \\
& \geqslant \log \left(\boldsymbol{w}_{i}^{T} \boldsymbol{p}^{*}\right), \quad \forall i, j .
\end{aligned}
$$

This is not a convex inequality, since the function on the right is a concave function in $\boldsymbol{p}^{*}$.

However, from Theorem 6.1 we can define $\log \left(p_{j}^{*}\right)=y_{j}^{*}$ for all $j$. Then, the above inequality becomes

$$
\begin{aligned}
& \log \left(\sigma_{i j}+a_{j}^{i} \bar{s}_{j}+\left(1-a_{j}^{i}\right) x_{i j}^{*}\right)+y_{j}^{*}+\log \left(\sum_{j=1}^{n} \frac{x_{i j}^{*}}{\sigma_{i j}+a_{j}^{i} \bar{s}_{j}+\left(1-a_{j}^{i}\right) x_{i j}^{*}}\right) \\
& \geqslant \log \left(\sum_{j} w_{i j} e^{y_{j}^{*}}\right), \quad \forall i, j .
\end{aligned}
$$

This is now a convex inequality, since the function on the left is a (strictly) concave function in $x_{i j}^{*}$ and $y_{j}^{*}$ for any constant $1-a_{j}^{i} \geqslant 0$, and the one on the right is a convex function in $y^{*}$. Therefore, we have the following.

Theorem 6.2 Under the weak-interference communication channel condition, the competitive equilibrium set of the spectrum trading market is log convex (convex in allocation and log price), and an equilibrium can be computed in polynomial time.

\section{Equilibrium Under the Frequency Division Multiple Access Policy}

For continuous communication spectrum management under the Frequency Division Multiple Access policy or even multiple users sharing the same tone but operating at different time point (that is, at any given time period, only one user utilizes the tone), each user's utility function is independent of other users, that is,

$$
u_{i}\left(\boldsymbol{x}_{i}\right)=\sum_{j=1}^{n} \log \left(1+\frac{x_{i j}}{\sigma_{i j}}\right) .
$$


Then, similar to the discussion in the previous section, one can rewrite the nonlinear inequality in (4.3) as

$$
\log \left(\sigma_{i j}+x_{i j}^{*}\right)+\log \left(p_{j}^{*}\right)+\log \left(\sum_{j=1}^{n} \frac{x_{i j}^{*}}{\sigma_{i j}+x_{i j}^{*}}\right) \geqslant \log \left(w_{i}\right), \quad \forall i, j ;
$$

or $(6.3)$ as

$$
\log \left(\sigma_{i j}+x_{i j}^{*}\right)+\log \left(p_{j}^{*}\right)+\log \left(\sum_{j=1}^{n} \frac{x_{i j}^{*}}{\sigma_{i j}+x_{i j}^{*}}\right) \geqslant \log \left(\boldsymbol{w}_{i}^{T} \boldsymbol{p}^{*}\right), \quad \forall i, j .
$$

Again, this is a convex or log-convex inequality. Therefore, we have the following.

Theorem 7.1 Under the FDMA or single-user-single-frequency at any time period policy, the competitive equilibrium of the discretized communication spectrum market or the spectrum trading market is unique, and it can be computed in polynomial time.

For a single frequency $j$ with multiple users $x_{i j}^{*}>0$, one can implement an online or real time policy on tone $j$ by a randomized algorithm by assigning user $i$ with probability $\frac{x_{i j}^{*}}{\sum_{k} x_{k j}^{*}}$ at any time period or when multiple users request services on tone $j$.

\section{An Illustration Example}

Consider two channels $f_{1}$ and $f_{2}$ and two users $\boldsymbol{x}$ and $\boldsymbol{y}$; each of them have a physical power budget $b_{1}=b_{2}=1$. Let the Shannon utility function for $\boldsymbol{x}$ be

$$
\log \left(1+\frac{x_{1}}{1+y_{1}}\right)+\log \left(1+\frac{x_{2}}{4+y_{2}}\right)
$$

and one for user $\boldsymbol{y}$ be

$$
\log \left(1+\frac{y_{1}}{2+x_{1}}\right)+\log \left(1+\frac{y_{2}}{4+x_{2}}\right)
$$

and let the aggregate social utility be the sum of the two individual user utilities.

Clearly, the Nash equilibrium is $x_{1}=y_{1}=1$ and $x_{2}=y_{2}=0$, where the social utility has value 0.3010 .

The cooperative social solution is $x_{1}=y_{2}=1$ and $x_{2}=y_{1}=0$, where the social utility has value 0.3979 , but it is not individually optimal for user $\boldsymbol{y}$.

Now consider a competitive spectrum market with power supply for two channels $s_{1}=s_{2}=b_{1}+b_{2}=2$ and the initial endowments for two users $w_{x}=w_{y}=1$. Then the unique competitive equilibrium point is

$$
\begin{array}{lll}
p_{1}^{*}=3 / 5 & \text { and } & p_{2}^{*}=2 / 5, \\
x_{1}^{*}=5 / 3 & \text { and } & x_{2}^{*}=0, \\
y_{1}^{*}=1 / 3 & \text { and } & y_{2}^{*}=2,
\end{array}
$$


with the social utility value 0.566 . However, the social utility value of the Nash equilibrium with the same physical power budget $b_{1}=5 / 3$ and $b_{2}=7 / 3$ has value 0.4657 .

Even we simply scale the spectrum allocation for the competitive market equilibrium to

$$
x_{1}^{*}=1, \quad x_{2}^{*}=0, \quad y_{1}^{*}=1 / 7, \quad y_{2}^{*}=6 / 7 .
$$

Such that each user is allocated exactly 1 unit physical power. Then, this allocation still has a social utility value 0.3775 that is much higher than that at the Nash equilibrium.

Furthermore, if we adjust the initial endowment to $w_{x}=6 / 5$ and $w_{y}=4 / 5$, then the equilibrium price will remain the same and the unique equilibrium allocation will be

$$
x_{1}^{*}=2, \quad x_{2}^{*}=0, \quad y_{1}^{*}=0, \quad y_{2}^{*}=2 .
$$

Upon scaling, we obtain the socially optimal allocation. In other words, the exogenous factors $\boldsymbol{w}$ and $\boldsymbol{s}$ of the spectrum market can be further adjusted to achieve a better social solution while maintaining individual satisfaction under the open market equilibrium price.

Now, consider the spectrum trading market where the initial power endowment for users $x$ and $y$ are given as

$$
\boldsymbol{w}_{x}=\frac{2}{3} \cdot\left(\begin{array}{l}
1 \\
1
\end{array}\right) \quad \text { and } \quad \boldsymbol{w}_{y}=\frac{1}{3} \cdot\left(\begin{array}{l}
1 \\
1
\end{array}\right),
$$

respectively. Thus, we have total one unit power in each tone in the trading market. The unique equilibrium point is

$$
\begin{array}{lll}
p_{1}^{*}=2 & \text { and } & p_{2}^{*}=1, \\
x_{1}^{*}=1 & \text { and } & x_{2}^{*}=0, \\
y_{1}^{*}=0 & \text { and } & y_{2}^{*}=1 .
\end{array}
$$

Basically, user $x$ has exchanged its $2 / 3$ unit power in tone 2 for $1 / 3$ unit power in tone 1 of user $y$. User $x$ 's utility value increased from 0.2382 to 0.3010 , and user $y$ 's utility value increased from 0.0811 to 0.0969 .

Acknowledgements The author thanks Tom Luo and Shuzhong Zhang for many insightful discussions on this subject.

Open Access This article is distributed under the terms of the Creative Commons Attribution License which permits any use, distribution, and reproduction in any medium, provided the original author(s) and the source are credited.

\section{References}

[1] Arrow, K.J., Debreu, G.: Existence of an equilibrium for a competitive economy. Econometrica 22(3), 265-290 (1954)

[2] Brainard, W.C., Scarf, H.: How to compute equilibrium prices in 1891. Cowles Foundation Discussion Paper 1270 (2000) 
[3] Cendrillon, R., Yu, W., Moonen, M., Verliden, J., Bostoen, T.: Optimal multi-user spectrum management for digital subscriber lines. IEEE Trans. Commun. 54(5), 922-933 (2006)

[4] Chan, V.M.K., Yu, W.: Joint multi-user detection and optimal spectrum balancing for digital subscriber lines. Eur. J. Appl. Signal Process. (EURASIP) 2006, Article ID 80941 (2006), pp. 1-13

[5] Chen, L., Ye, Y., Zhang, J.: A note on equilibrium pricing as convex optimization. Int. Netw. Econ. Lect. Notes Comput. Sci. 4858, 7-16 (2007). Springer, Berlin

[6] Chen, N., Deng, X., Sun, X., Yao, A.C.: Fisher equilibrium price with a class of concave utility functions. In: Proceedings of ESA 2004, pp. 169-179 (2004)

[7] Chung, S.T., Kim, S.J., Lee, J., Cioffi, J.M.: A game-theoretic approach to power allocation in frequency-selective Gaussian interference channels. In: Proceeding 2003 IEEE International Symposium on Information Theory, Yokohama, Japan (2003)

[8] Codenotti, B., Saberi, A., Varadarajan, K., Ye, Y.: Leontief economies encode nonzero sum twoplayer games. In: Proc. SODA’06, pp. 659-667 (2006)

[9] Cover, T.M., Thomas, J.A.: Elements of Information Theory. Wiley, New York (1991)

[10] Debreu, G.: A social equilibrium existence theorem. Proc. Natl. Acad. Sci. 38, 886-893 (1952)

[11] Deng, X., Huang, S., Ye, Y.: Computation of the Arrow-Debreu equilibrium for a class of nonhomogenous utility functions. Technical Report, State Key Laboratory of Intelligent Technology and Systems, Dept. of Computer Science and Technology, Tsinghua Univ., Beijing, 10084, China

[12] Eisenberg, E., Gale, D.: Consensus of subjective probabilities: the Pari-Mutuel method. Ann. Math. Stat. 30, 165-168 (1959)

[13] Eisenberg, E.: Aggregation of utility functions. Manag. Sci. 7(4), 337-350 (1961)

[14] Gale, D.: The Theory of Linear Economic Models. McGraw Hill, New York (1960)

[15] Haykin, S.: Cognitive radio: brain-empowered wireless communications. IEEE J. Sel. Areas Commun. 23, 201-220 (2005)

[16] Jain, K., Vazirani, V., Ye, Y.: Market equilibria for homothetic, quasi-concave utilities and economies of scale in production. In: Proc. SODA'05, pp. 63-71 (2005)

[17] Lui, R., Yu, W.: Low complexity near optimal spectrum balancing for digital subscriber lines. In: IEEE International Conference on Communications (ICC), Seoul, Korea, vol. 3, pp. 1947-1951 (2005)

[18] Luo, Z.-Q., Pang, J.-S.: Analysis of iterative water-filling algorithm for multi-user power control in digital subscriber lines. Special issue of EURASIP. J. Appl. Signal Process. Adv. Signal Process. Tech. Digit. Subscr. Lines 2006, Article ID 24012 (2006), 10 pp.

[19] Luo, Z.-Q., Zhang, S.: Dynamic spectrum management: complexity and duality. IEEE J. Sel. Top. Signal Process. 2(1), 57-73 (2008)

[20] Luo, Z.-Q., Zhang, S.: Duality gap estimation and polynomial time approximation for optimal spectrum management. IEEE Trans. Signal Process. 57(7), 2675-2689 (2009)

[21] Nash, J.F. Jr.: Equilibrium points in X-person games. Proc. Natl. Acad. Sci. 36, $48-49$ (1950)

[22] Scarf, H.E.: The Computation of Economic Equilibria. Yale University Press, New Haven (1973). With collaboration of T. Hansen, Cowles Foundation Monograph No. 24

[23] Song, K.B., Chung, S.T., Ginis, G., Cioffi, J.M.: Dynamic spectrum management for next-generation DSL systems. IEEE Commun. Mag. 40, 10-110 (2002)

[24] Walras, L.: Elements d'economie politique pure; ou, Theorie de la richesse sociale. In: Elements of Pure Economics; Or the Theory of Social Wealth, Lausanne, Paris (1874)

[25] Yamashita, N., Luo, Z.-Q.: A nonlinear complementarity approach to multi-user power control for digital subscriber lines. Optim. Methods Softw. 19, 633-652 (2004)

[26] Ye, Y.: A path to the Arrow-Debreu competitive market equilibrium. Math. Program. Ser. B 111(12), 315-348 (2008)

[27] Ye, Y.: Exchange market equilibria with Leontief's utility: freedom of pricing leads to rationality. Theor. Comput. Sci. 378(2), 134-142 (2007)

[28] Yu, W., Ginis, G., Cioffi, J.M.: Distributed multi-user power control for digital subscriber lines. IEEE J. Sel. Areas Commun. 20, 1105-1115 (2002)

[29] Yu, W., Lui, R.: Dual methods for nonconvex spectrum optimization of multicarrier systems. IEEE Trans. Commun. 54, 1310-1322 (2006)

[30] Yu, W., Lui, R., Cendrillon, R.: Dual optimization methods for multi-user orthogonal frequency division multiplex systems. In: IEEE Global Communications Conference (Globecom), Dallas, USA, vol. 1, pp. 225-229 (2004) 